\title{
CONTRACEPCIÓN Y FEMINIDADES: UN ANÁLISIS DE LA RESISTENCIA A LA PASTILLA
}

\section{CONTRACEPTION AND FEMININITIES: AN ANALYSIS OF THE RESISTANCE TO THE PILL}

\section{Tania Cuevas Barberousse*}

RESUMEN

\begin{abstract}
Este artículo analiza las razones por las cuales un grupo de mujeres abandona el uso de la pastilla anticonceptiva. El artículo evidencia que esta decisión puede pensarse como una resistencia a ciertas representaciones, valores y prácticas inscritas en la pastilla. En ese sentido, la pastilla es considerada como un guión de género. El análisis de las experiencias del grupo de mujeres estudiado revela que en ciertas condiciones, la noción de la pastilla anticonceptiva como una tecnología intrínsecamente emancipadora, puede ser cuestionada.
\end{abstract}

PALABRAS CLAVE: CONTRACEPCIÓN * FEMINIDAD * GÉNERO * RESISTENCIA

\section{ABSTRACT}

This article analyses why a group of women abandoned the contraceptive pill. It argues that this decision can be analyzed as a form of resistance to certain representations, values, and practices inscribed in the pill. In this case, the contraceptive pill is considered as a gender script. The study of the experiences of a group of women reveals that, under certain conditions, the notion of the contraceptive pill as an intrinsically emancipatory technology can be challenged.

KEYWORDS: CONTRACEPTION * FEMININITY * GENDER * RESISTANCE 


\section{INTRODUCCIÓN}

Actualmente, la pastilla anticonceptiva es consumida por más de 100 millones de mujeres alrededor del mundo (Department of Economics and Social Affairs, 2012). Sin embargo, $y$ a pesar de su popularidad, algunas mujeres deciden por voluntad propia, abandonar el uso de esta tecnología. En este artículo interesa explorar y analizar las razones que motivan a un grupo de mujeres a dejar de consumir la pastilla, así como, las percepciones y significaciones simbólicas que se construyen alrededor de esta tecnología contraceptiva $y$ las identidades de género de estas mujeres. Al mismo tiempo, este artículo examina las estructuras de poder desde las cuales se produce esta tecnología y los discursos que se promueven acerca de los cuerpos femeninos. El objetivo es por lo tanto, analizar los roles sexuales y la dominación que este tipo de tecnologías supone y reproduce. El hecho de no utilizar la pastilla, ¿ilustra formas de resistencia a los discursos dominantes acerca de los cuerpos, la sexualidad y la reproducción femeninos?

Se considera que el análisis del uso y de la resistencia a las tecnologías contraceptivas es necesario para comprender la manera en que se (re)configuran ciertas formas de identidades de género, así como, la manera en que ciertas mujeres (re)negocian ciertas prácticas y discursos biomédicos. Desde el punto de visto teórico, este artículo se inscribe en los estudios sociales de la ciencia y la tecnología (Science and Technology Studies, sTS), los cuales sugieren que la pastilla puede ser pensada como un script, es decir, como una inscripción o un guión de género. Así, este artículo busca responder a las siguientes preguntas: ¿cuáles son las identidades de género inscritas en la pastilla?, ¿qué aspectos de ese guión son resistidos (o negociados) por las mujeres que abandonan su consumo?, ¿cuáles son los mecanismos utilizados por estas mujeres para resistir ese guión? y ¿qué consecuencias tiene esa resistencia?

\section{DISCUSIÓN TEÓRICA}

Esta sección tiene como objetivo presentar las diversas perspectivas teóricas que a juicio personal, permiten reflexionar acerca del consumo de la pastilla anticonceptiva. Se parte de la idea de que la pastilla anticonceptiva puede pensarse como un guión de género, es decir, como una tecnología que materializa representaciones de género particulares, así como, valores y prácticas que reposan sobre estructuras sociales y culturales determinadas. Oudshoorn (2003) define la pastilla en términos foucaldianos, es decir, como una tecnología disciplinaria que forma parte de la socialización y la normalización del comportamiento reproductivo.

La conceptualización del género como una "performatividad", tal y como lo propone Judith Butler (1990 y 1993), parece útil para entender este enfoque. Para la autora, ni la tecnología ni el género poseen cualidades intrínsecas. En ese sentido, el género no es algo que es, sino algo que se hace. Inspirándose en las tesis del filósofo J.L. Austin, Butler desarrolla de esta manera una crítica radical a la noción de género como una categoría fija anclada en la naturaleza o los cuerpos. Esta posición postestructuralista considera al género como el resultado de prácticas discursivas que tienen el potencial de producir lo que nombran. Esta teoría se inserta en una matriz de poder heterosexual $y$ heteronormativo que supone el mantenimiento de convenciones de género muy particulares.

Para Oudshoorn (2003), analizar en qué medida las tecnologías contraceptivas contribuyen al mantenimiento o a la transformación de ciertas performatividades de género, es una cuestión importante, ya que estas tecnologías estudiadas en tanto actores no humanos, pueden ayudar a entender los procesos implicados en la estabilización o la desestabilización de convenciones particulares de género. Según este punto de vista, el género y la tecnología se conciben como mutuamente constituidos y co-producidos (Oudshoorn, 2003). El predominio de métodos contraceptivos para mujeres contribuyó, por ejemplo, a la estabilización de ciertas performatividades de género. Así, el énfasis puesto en la construcción social y cultural de la pastilla, permite entender no solamente las instituciones sociales y las prácticas 
implicadas en la construcción de esa tecnología, sino también las significaciones simbólicas, formación de identidades y sistemas de creencia que ella supone.

En ese sentido, el rol de la Biología y de la Medicina en la producción de lo masculino y de lo femenino, $y$ sus representaciones en tanto categorías naturales, ahistóricas y asociales, es fundamental para aprehender las implicaciones del consumo $y$ de la resistencia a la pastilla. Oudshoorn sostiene que la pastilla anticonceptiva puede ser considerada como el resultado más exitoso de lo que ella denomina como el concepto del "cuerpo hormonal" (1994: 9). Para la autora, aunque hoy es casi imposible imaginarse un mundo sin hormonas, el concepto de hormona $y$ de hormona sexual en particular, es muy reciente. Hasta el siglo xIx, por ejemplo, las mujeres no utilizaban esos términos para referirse a sus experiencias corporales. En efecto, tal concepto hizo su aparición hacia inicios del siglo xx.

El estudio de las "secreciones internas" del cuerpo o endocrinología, emergió como un novedoso campo de investigación hacia finales del siglo xix. Intentando encontrar un modelo explicativo alternativo a la teoría del estímulo nervioso (según la cual, el sistema nervioso era el responsable de todos los procesos fisiológicos del cuerpo), los científicos de la época se dieron a la tarea de buscar y testar todas las sustancias químicas secretadas por el cuerpo. A esas sustancias químicas se les denominó como "hormonas". El primer científico en utilizar tal concepto fue el profesor de fisiología británico Ernest H. Starling, en 1905. Estas nuevas sustancias se convirtieron rápidamente en una nueva línea de investigación. La idea de que las hormonas fueran capaces de controlar procesos fisiológicos sin la mediación del tejido nervioso, era particularmente innovadora y atrayente.

Para Oudshoorn (1994), el concepto de hormona se convirtió así en un hecho científico y universal. Además supuso un conjunto de transformaciones en la comprensión y la interpretación de los cuerpos, especialmente el de las mujeres. Según la autora, los procesos a través de los cuales las afirmaciones científicas alcanzan su estatus de hechos naturales y universales, permiten cuestionar la idea de que la ciencia revela la "verdad" de la naturaleza. En ese sentido, Oudshoorn propone una visión epistemológica que intenta ir más allá de la imagen tradicional de la ciencia, sugiriendo que los científicos descubren la realidad pero al mismo tiempo, crean esa realidad. Este enfoque constructivista supone que las hormonas no solo fueron descubiertas, sino que fueron creadas literalmente, por los científicos. Oudshoorn afirma que lo que le confiere poder a la ciencia es su capacidad de (re)crear nuevos objetos y nuevos universos. De esa manera, la autora afirma que las ciencias establecen una autoridad material que es predominante en nuestra cultura. En el caso de la endocrinología, su autoridad material se hizo posible en el momento en el cual el concepto teórico de hormona se transformó en realidad tangible, es decir, en nuevas sustancias químicas y en nueva familia de medicamentos.

Para Clarke (1998), la endocrinología y las ciencias reproductivas en general, son probablemente las disciplinas que construyen y mantienen de forma más marcada, los discursos y las prácticas relativas al género y al sexo. En el caso de la endocrinología, considera la autora, para que los hechos científicos propuestos por esta nueva disciplina fueran considerados como tales, adquirieran su forma definitiva y su estatus de verdad universal, los endocrinólogos debieron crear alianzas con grupos de actores externos a la disciplina. En ese sentido, los estudios de la ciencia y la tecnología recurren a la noción de red para conceptualizar la manera en que los hechos científicos y las tecnologías - en este caso, el concepto de hormona y la pastilla anticonceptiva - adquirieron validez en un proceso interactivo entre distintos actores sociales. Este concepto, formulado por Pinch y Bijker (1987), provee un modelo para entender hasta qué punto los hechos científicos se establecen como tales, solo si logran articularse con los intereses institucionales y las prácticas de otros grupos de actores $y$ sus audiencias. De esta manera, se puede considerar a estos grupos como comunidades que comparten a la vez rasgos discursivos y prácticos. Esta conceptualización de la ciencia 
permite analizar las razones por las cuales ciertas afirmaciones sobre las hormonas y el cuerpo femenino (no sobre el cuerpo masculino), por ejemplo, se convirtieron en hechos universales. Así, la investigación sobre las hormonas se efectuó dentro de una red formada por tres grupos de actores, a saber: los endocrinólogos, los ginecólogos y la industria farmacéutica.

Según Oudshoorn (1994), es en este contexto que las sustancias provenientes de las glándulas sexuales fueron designadas como las hormonas sexuales: las hormonas sexuales masculinas, secretadas por los testículos y las hormonas sexuales femeninas, secretadas por los ovarios. Una vez establecida, esta conceptualización de las hormonas creó una dualidad sexual (Oudshoorn, 1990). La noción de las hormonas como los agentes de la masculinidad $y$ de la feminidad de los cuerpos funcionó como un paradigma. Así, todas las hipótesis propuestas se fundamentaron en esta noción de dualidad y la diferenciación sexual se construyó sobre la base de ideas preconcebidas sobre la masculinidad $y$ la feminidad. Tal $y$ como lo demuestra Sinding (2003), a pesar del hecho de que los científicos comenzaron a percatarse de ciertos efectos considerados como "femeninos" de las hormonas masculinas y de ciertos efectos considerados como "masculinos" de las hormonas femeninas, estos prefirieron cuestionar la sexualidad de los sujetos testados antes que alterar el "modelo de los dos sexos" (Laqueur, 1990: 8).

Ahora bien, la introducción del concepto de hormonas sexuales no solo implicó una serie de transformaciones en los estudios del cuerpo, sino también y probablemente más importante, en su construcción e interpretación (Oudshoorn, 1994). Desde esta perspectiva, diversos tipos de comportamientos, roles, funciones y características considerados como esencialmente femeninos o masculinos, comenzaron a ser atribuidos a las hormonas. En ese sentido, la introducción del concepto de hormonas sexuales como mensajeras químicas de la masculinidad y de la feminidad, implicó una transformación radical del concepto de sexo. En efecto, éste se concibió no solo como el resultado de características anatómicas sino también como el resultado de sustancias químicas. Así, lo que Oudshoorn denomina como el concepto del cuerpo hormonal permitió la construcción de nuevas significaciones y prácticas asociadas al cuerpo humano.

Las hormonas sexuales funcionaron por lo tanto como agentes dobles: por un lado, como herramientas terapéuticas, pero por otro, como herramientas de control y de regulación de los cuerpos individuales y sociales. Para Oudshoorn (1990), el concepto de hormonas permitió a los ginecólogos una mejor comprensión, pero sobre todo, un mejor control de los múltiples "desórdenes" asociados a los ovarios. En ese sentido, al asociar los "desórdenes femeninos" con las hormonas femeninas, los problemas "de las mujeres" se convirtieron en el dominio casi exclusivo de los ginecólogos (Moscucci, 1990). La pastilla anticonceptiva fue la mayor realización en ese sentido. Es importante resaltar que en ese contexto, el cuerpo femenino ( $y$ no el cuerpo masculino), fue representado como un cuerpo controlado por las hormonas y sobre todo, como un cuerpo que debía ser controlado. Este proceso de "hormonización" se sustentó en una naturalización del cuerpo de las mujeres ${ }^{1}$.

La pastilla fue producida en Estados Unidos hacia el final de los años 40 y principios de los años 50, y constituyó una novedad en el mercado de los métodos contraceptivos. Para Clarke (1998), el desarrollo y la introducción de los métodos de contracepción modernos ${ }^{2}$ son el

1 Para un análisis más detallado del proceso de naturalización de los cuerpos femeninos, ver Cuevas, 2013.

2 Según Clarke (1998), hasta los años 60, los métodos contraceptivos utilizados eran lo que ella denomina como dispositivos "simples", es decir, tecnologías relativamente fáciles de utilizar y que no necesitaban de una intervención médica específica, como por ejemplo, los métodos de barrera, los espermicidas, las duchas vaginales, el método del ritmo y el uso de hierbas medicinales. En contraste, la pastilla anticonceptiva pasó a formar parte de los "métodos científicos modernos", es decir, métodos que como consecuencia del desarrollo de disciplinas como la endocrinología, son tecnológicamente más sofisticados, sistémicos y cuyo consumo requiere de la pericia médica. Los métodos "simples" van a ir perdiendo legitimidad frente 
resultado de una empresa moderna por excelencia, a saber, el control del cuerpo femenino y de la reproducción. En efecto, ella argumenta que la modernidad se ha caracterizado por esfuerzos continuos por racionalizar e industrializar una amplia variedad de procesos fisiológicos. El control de la reproducción siguió una lógica del fordismo basada en la racionalización de los procesos reproductivos, la producción y la distribución de nuevos productos y la creación de tecnologías, servicios y cuidados que vinieron a reforzar ese control. Como consecuencia, Clarke afirma que la reproducción fue disciplinada, no solo en el sentido en que un poder fue ejercido sobre el momento, los medios $y$ la frecuencia de la concepción (evocando la noción de bio-poder de Foucault), sino también en el sentido en que hubo una formación disciplinaria (la endocrinología) que permitió toda una serie de desarrollos tecnológicos, sin los cuales la pastilla no pudo haber sido creada. En mayo de 1960, la Food and Drug Administration (FDA) aprobó su comercialización bajo el nombre de Enovid. En 1965, solo cinco años después de su introducción al mercado, la pastilla anticonceptiva era el método más utilizado en Estados Unidos (Engelman, 2011). Más adelante, la pastilla se convirtió en la sustancia farmacológica más vendida de la historia (Oudshoorn, 1994).

Se considera que el consumo de la pastilla anticonceptiva representa un espacio idóneo para entender ideas clave acerca de la feminidad y el cuerpo femenino, como por ejemplo, su medicalización, su "ciclicidad"3 y su función primordialmente reproductora. Sin embargo, van Oost (2003) advierte que la noción de guión no debe confundirse con la idea de determinación, aunque invita a aceptar las ideas inscritas en la tecnología, en este

a estos métodos "modernos", considerados como más "técnicos" y por lo tanto, más "científicos" (Tone, 1999).

3 El análisis de los procesos de la sexuación del cuerpo femenino en la medicina moderna y contemporánea, ha señalado el papel de la teoría hormonal y la construcción, a comienzos del siglo $\mathrm{xx}$, de la idea de "ciclicidad" química (femenina) como opuesta a la estabilidad masculina. Para un análisis más detallado, ver Ortiz, 2004. caso, la pastilla, los guiones pueden ser negociados o incluso, resistidos.

En ese sentido, diversas investigaciones sobre el tema de la resistencia y el no uso de la tecnología, ilustran la importancia de estos procesos de negociación (Kline, 2003 y Wyatt, 2003). Estos estudios sugieren que los usuarios no son simples recipientes pasivos de la tecnología, sino que en sus prácticas de consumo o resistencia, negocian constantemente, tanto sus propias identidades como el de las tecnologías. Diversas inquietudes surgen de esta línea de investigación con respecto al tema de las identidades de género. Aunque la pastilla anticonceptiva es consumida actualmente por millones de mujeres alrededor del mundo, un grupo importante de ellas elige por voluntad propia, no consumirla. ¿Qué significado tiene esta resistencia?, ¿puede pensarse este rechazo como una forma de negociar discursos dominantes acerca de la sexualidad, la reproducción $y$ el cuerpo femenino inscrito en la pastilla?

\section{METODOLOGÍA}

Esta investigación busca contestar estas preguntas por medio del análisis de las razones que llevaron a un grupo de mujeres a no consumir la pastilla anticonceptiva y de las formas en que experimentaron $y$ vivieron esa resistencia. Desde el punto de vista metodológico, este artículo se basa en una etnografía virtual de un foro en Internet dedicado a la discusión del uso de la pastilla anticonceptiva: Aphrodite (En: $<\mathrm{http}$ ://www.aphroditewomenshealth.com $>)^{4}$.

Hine (2000) propone que la etnografía virtual es una aproximación metodológica que consiste en examinar los textos y discursos producidos en Internet, con el propósito de entender dinámicas generales de producción de significado. Con los calificativos de "virtual" o "digital", la etnografía se ha convertido en un

$4 \quad$ Se decidió analizar el foro Aphrodite, después de realizar una búsqueda en Internet de foros dedicados a la discusión sobre la sexualidad y la contracepción femeninas. Con miles de usuarias, Aphrodite es uno de los foros más organizado y frecuentado. El idioma utilizado es el inglés y las normas de interacción entre las usuarias están muy reglamentadas. 
método privilegiado para analizar las "culturas" en el contexto de Internet, así como, las prácticas ligadas a su utilización (Boellstorff, 2008; Burrell, 2009 y Coleman, 2010). En ese sentido, Internet constituye el terrero en el cual se lleva a cabo la etnografía y al mismo tiempo, la tecnología a partir de la cual se efectúa.

Si bien, las primeras investigaciones sobre el mundo "virtual" tendían a concebir Internet como un espacio social diferente al "mundo real", investigaciones más recientes demuestran la imposibilidad de separar ambos mundos (Orgad, 2005 y Rogers, 2013). Así, la "realidad virtual" no se concibe como una entidad separada de otros aspectos de la acción $y$ de la experiencia humana. En ese sentido, lo que interesa es examinar cómo el uso de Internet facilita la expresión de concepciones culturales más amplias acerca de la feminidad. Por lo tanto, se trata de decodificar discursos, prácticas $y$ actitudes de un grupo de mujeres miembras de un foro en Internet, con respecto al uso de la pastilla (Thomsen, Straubhaar y Bolyard, 1998).

Diversas razones justificaron esta decisión metodológica. Primero, Internet se ha convertido en uno de los medios más importantes de acceso y circulación de información relacionada con la salud (Akrich y Méadel, 2004). En el caso de la pastilla anticonceptiva, Internet representa un espacio ideal para estudiar las actitudes y prácticas de mujeres con respecto a su consumo. Los foros de discusión, en particular, brindan acceso directo a las interacciones en línea de la población que interesa, a saber, grupos de mujeres que discuten en torno al consumo o no de la pastilla $y$ de esa forma, permiten recoger datos que de otra forma se encontrarían dispersos. Además, los foros en Internet son utilizados no solamente para consultar información, sino también para conocer y compartir experiencias. Al consultar los archivos de estos foros, es posible recuperar esas experiencias para un análisis de los significados y percepciones asociados a la pastilla. Finalmente, Internet da la posibilidad de comparar información producida en contextos sociales y culturales distintos, por lo tanto, permite comparar las vivencias de diversas mujeres.

Para efectos de este artículo, el enfoque utilizado por Walstrom (2004) en su estudio de grupos de apoyo en línea, pareció el más apropiado. En este, la autora utiliza el término de "participante-experimentadora" en lugar del de "participante-observadora" para caracterizar la naturaleza del rol del investigador en el contexto de Internet. En el caso del artículo, se sigue la misma estrategia.

Por lo tanto, procedí a suscribirme al foro Aphrodite, el día 11 de octubre de 2011. En este sitio me presenté como investigadora $y$ consumidora de la pastilla $y$ después de compartir mi experiencia personal, así como de leer $y$ analizar los mensajes publicados por las usuarias de este foro, publiqué 7 preguntas (en inglés) con el fin de conocer y examinar las vivencias de este grupo de mujeres miembras del foro con respecto a la pastilla anticonceptiva. Estas preguntas fueron respondidas por 19 de esas mujeres, entre enero y mayo de 2012. Además, se realizaron 12 entrevistas a profundidad con el fin de analizar, de manera más detallada, la construcción de sus identidades de género y las dinámicas de negociación y de resistencia en relación a las tecnologías contraceptivas. Estas entrevistas se llevaron a cabo entre enero y mayo de 2012. Fueron realizadas de manera asincrónica $y$ en inglés, ya sea por correo electrónico o por el sistema de mensajería privada del foro de discusión. Con el fin de respetar las identidades de las mujeres encuestadas, se decidió no utilizar sus nombres reales $y$ sustituirlos por nombres ficticios.

\section{RESULTADOS}

\section{LAS EXPERIENCIAS CON LA PASTILLA}

\subsection{UNA FEMINIDAD CÍCLICA}

Esta sección tiene como objetivo poner en evidencia las razones por las cuales las mujeres encuestadas comenzaron a consumir la pastilla y el rol de los médicos en esta toma de decisión. Las respuestas revelan dos elementos interesantes: primero, que además de su función contraceptiva, la pastilla es muchas 
veces prescrita como medio para controlar ciertos procesos fisiológicos y segundo, que el rol de los médicos en la promoción de los contraceptivos hormonales aparece como central. En efecto, para la mayoría de las mujeres analizadas, la principal razón para consumir la pastilla no fue la de evitar un embarazo, sino la de regular y controlar sus ciclos menstruales, así como, los síntomas asociados a la menstruación. Este punto parece importante en la medida en que esta voluntad de intervención permite evidenciar las condiciones en las cuales ciertos procesos contemporáneos de biomedicalización participan en la transformación de la corporalidad femenina. De las 19 mujeres encuestadas, solamente 4 respondieron haber comenzado a utilizar la pastilla por razones estrictamente contraceptivas. Amalia, una diseñadora gráfica de 26 años de New Jersey, Estados Unidos, afirmó: "para mí la pastilla era lo más inteligente $y$ responsable porque eso es lo que la sociedad nos dice a nosotras las mujeres. Cuando fui a ver a mi ginecóloga, ella me prescribió inmediatamente Yaz, la pastilla más popular en aquella época". Lo interesante de resaltar aquí es la idea de que la contracepción es una responsabilidad esencialmente femenina.

Entre las 15 mujeres restantes, 6 declararon haber comenzado a utilizar la pastilla como método contraceptivo y como método para regular sus ciclos menstruales. Violeta, una ama de casa inglesa de 27 años comentó: "yo utilizaba la pastilla como método contraceptivo, pero también porque estaba frustrada con la irregularidad de mis sangrados. Después de mi primer hijo, mi partera prácticamente me insistió para que tomara la pastilla $y$ así poder tener una especie de ciclo". Lo importante de señalar en este caso es el rol protagonista de los médicos y los profesionales de la salud en general, en la promoción de los contraceptivos hormonales como medios para regular $y$ controlar los ciclos menstruales de las mujeres. Gabriela, una oficial de seguridad de 25 años que vive en Canadá, afirmó que comenzó a utilizar la pastilla porque "mi médico me dijo que me iba a ayudar a regular mis ciclos que eran muy dolorosos y abundantes. En esa época no era sexualmente activa".
Es interesante resaltar que un ciclo menstrual regular parece ser un objetivo deseado, tanto por las mujeres encuestadas como por sus médicos. La menstruación, la mayoría de las veces descrita como "abundante", "irregular" y "dolorosa", es vista como un proceso que debe ser "medicalizado" con el fin de lograr un funcionamiento "normal" del cuerpo. En ese sentido, Oudshoorn (1994) considera que la metáfora de la ciclicidad de los procesos fisiológicos femeninos, lejos de ser natural fue creada, literalmente, por los científicos. En efecto, la autora constata que durante los ensayos clínicos de la pastilla llevados a cabo en Puerto Rico y Haití, a inicios de la década de los 50 , los científicos "borraron" a las mujeres participantes, reemplazándolas por la categoría de "ciclos menstruales". Esta estrategia les permitió no solamente amplificar los resultados ${ }^{6}$, sino también subrayar las similitudes entre las mujeres. Estas similitudes no fueron solo una cuestión de discurso.

En efecto, Gregory Pincus, considerado como el "padre" de la pastilla, ante la angustia que representaba para las mujeres la interrupción de la menstruación como consecuencia del consumo de la pastilla (porque al suprimir la ovulación no hay reglas "naturales"), decidió "imitar" un ciclo menstrual: la pastilla debía ser tomada por 24 días después de los cuales seguirían 5 días de "reglas" para un total de 28 días. Tal y como el mismo Pincus lo indicó, el ciclo pudo haber tenido cualquier duración (Pincus, 1959). En consecuencia, la variabilidad de los ciclos menstruales femeninos fue anulada en la medida en que todas las usuarias de pastillas anticonceptivas debían tener un ciclo de cuatro semanas. Para Oudshoorn (1994), este énfasis en las similitudes entre las mujeres, era fundamental para producir una tecnología universal, es decir, un contraceptivo destinado a todas las mujeres. Es importante resaltar que

$5 \quad$ Es decir, la menstruación se convierte en un problema médico.

6 Por ejemplo, en un ensayo efectuado en Río Piedras, Puerto Rico, Pincus concluyó: "830 sujetos tomaron el medicamento, para un total de 8133 ciclos menstruales, o 635 años mujeres" (Pincus, 1959: 81). 
la representación en términos de "ciclos", implica la transformación del cuerpo de las mujeres en una categoría universal como consecuencia de un proceso fisiológico. La ecuación entre ciclicidad y feminidad le propuso a la medicina diferentes formas de controlar el cuerpo de las mujeres: acortando, alargando, pero generalmente, estandarizando los ciclos menstruales femeninos. En ese sentido, la medicalización del cuerpo de las mujeres influencia no solamente las formas en que las mujeres interpretan y perciben sus cuerpos, sino también la manera en que actúan los profesionales que trabajan en el sector de la salud.

Si bien, las mujeres encuestadas no ponen en entredicho a la pastilla, en tanto método para regular sus ciclos menstruales, si cuestionan otros guiones inscritos en la pastilla, como se verá a continuación.

\subsection{EL PODER DE LAS HORMONAS}

Esta sección tiene como objetivo examinar las razones por las cuales las mujeres encuestadas decidieron abandonar el uso de la pastilla anticonceptiva. Tal y como se demostró en el apartado anterior, si bien estas mujeres comenzaron a consumir la pastilla para mejorar sus vidas de manera general (ya sea controlando sus ciclos o evitando un embarazo no deseado), la mayoría de ellas abandonó su utilización por diversas razones. Lo que resulta interesante de resaltar es que la evaluación hecha por las mujeres de los efectos secundarios producidos por la pastilla no se limitó a la descripción de los efectos físicos que ellas asociaron a su consumo, sino también y sobre todo, a los efectos de orden psicológico y emocional.

En el caso de los métodos contraceptivos como la pastilla, los efectos secundarios se vuelven problemáticos en la medida en que las usuarias, son a priori, mujeres saludables. Esta especificidad de la pastilla plantea complicaciones para las mujeres que consideran que sus efectos secundarios son más problemáticos que sus posibles beneficios. En el caso que se analiza, de las 19 mujeres encuestadas, 17 decidieron no utilizar más la pastilla anticonceptiva. Laura, una estudiante canadiense de 19 años comentó: "yo dejé la pastilla porque me di cuenta que me estaba deprimiendo. Estoy segura que la palabra que usé cuando fui a ver a mi ginecólogo fue "bipolar". Antes mis amigos me describían como "una de las chicas más felices". Por eso decidí no volver a tomar nunca más la pastilla". Para Laura, los efectos provocados por la pastilla son de orden emocional. El uso del término "bipolar" sugiere que ella percibe una deterioración de su salud mental. Para ella, la toma de la pastilla significó una verdadera transformación de su personalidad. Esta descripción de síntomas emocionales y psicológicos es generalizado entre las mujeres encuestadas. Victoria, por ejemplo, una banquera californiana de 27 años, pone en relieve la depresión y la ansiedad que según ella, resultaron del consumo de la pastilla: "tomé la pastilla durante 5 años. Creo que mis constantes cambios de humor provocaron la ruptura de una relación casi perfecta. La pastilla me afectó mucho en términos de depresión y ansiedad. Viví los peores años de mi vida".

Lo que describe Victoria es el poder que ella le atribuye a las hormonas. En ese sentido, las hormonas son interpretadas como capaces de ejercer un control sobre la vida de las mujeres. Si se considera que las mujeres encuestadas comenzaron a consumir la pastilla para controlar sus cuerpos (su fertilidad o sus ciclos menstruales), los efectos secundarios desencadenados por la pastilla pueden ser vividos como profundamente contradictorios. Lo que se puede resaltar de las experiencias de las mujeres encuestadas, es el hecho de que los problemas provocados por la pastilla parecen tener consecuencias profundas sobre sus vidas cotidianas. En ese sentido, sus experiencias subrayan hasta qué punto los efectos secundarios provocados por la pastilla son interpretados como obstáculos para su bienestar. Pero esos efectos también pueden implicar riesgos graves para la salud de estas mujeres, como en el caso de Gabriela: "yo quería tener ciclos regulares. Mi ginecóloga me prescribió la pastilla. Tres semanas después, noté una vena muy roja e hinchada en mi entrepierna. Me dolía mucho. Fui a emergencias y me diagnosticaron una tromboflebitis. Los doctores me dijeron que había sido provocada por la pastilla". La experiencia de Gabriela permite reflexionar en un elemento fundamental para comprender 
e interpretar las experiencias de estas mujeres: que la responsabilidad y los riesgos asociados a la contracepción han sido históricamente delegados a las mujeres. Esta distribución desigual de los riesgos es un guión claramente materializado en la pastilla y es cuestionado por la mayoría de las mujeres encuestadas. Violeta, por ejemplo, prefirió asumir el riesgo de quedar embarazada en lugar de volver a tomar la pastilla: "estaba muy deprimida, y me dolía mucho la cabeza. Seguí tomándola porque honestamente hay tan pocas opciones pero llegué al punto de preferir quedar embarazada antes que seguir soportando los cambios de humor, los dolores en el pecho, la libido por el suelo, la depresión y la ansiedad".

Para la mayoría de las mujeres encuestadas, los efectos secundarios producidos por la pastilla se volvieron más problemáticos que sus posibles efectos contraceptivos. Este hecho desencadena una resistencia a ciertos aspectos del guión de género de la pastilla. Amalia, por ejemplo, afirmó: "yo estoy 100\% en contra de la pastilla o de cualquier método contraceptivo hormonal. No puedo imaginarme revivir el infierno que pasé". Por su parte, Katia, una estudiante de maestría de 30 años, residente en Miami, Estados Unidos, comentó: "jamás volvería a usar la pastilla. Me robó 10 años de mi vida”. Así, de las 19 mujeres encuestadas, 17 afirmaron que no volverían a consumir la pastilla o a utilizar otro método contraceptivo hormonal.

Esta resistencia a la pastilla se manifiesta más claramente cuando otras dimensiones del guión de género materializado en esta tecnología se integran en lo que llamaremos aquí como una red de relaciones sociales compuesta por sus ginecólogos, sus compañeros sentimentales y las otras mujeres del foro Aphrodite. A continuación se analizarán las tensiones, las negociaciones $y$ las alternativas que emergen en el seno de estos tres espacios.

\section{TENSIONES, NEGOCIACIONES Y ALTERNATIVAS}

\section{1. "NO ESTOY LOCA"}

Uno de los mecanismos utilizados por las mujeres encuestadas para hacer frente a los problemas causados por la pastilla, es el de hablar con sus médicos ginecólogos. Sin embargo, la relación establecida entre estos últimos y las encuestadas se revela como problemática por varias razones. Primero, en la actualidad la contracepción es esencialmente médica y esto supone una relación muy particular entre las mujeres y los médicos (Toulemon y Leridon, 1991). Segundo, porque las mujeres encuestadas no encontraron ni el alivio ni el apoyo esperado por parte de sus médicos, lo que provocó una insatisfacción generalizada, así como, tensiones importantes.

En efecto, uno de los aspectos más criticados por las mujeres fue lo que ellas consideraron como una promoción constante de los contraceptivos hormonales por parte de los ginecólogos. Alicia, una estudiante de 23 años de Texas, Estados Unidos y María, una maestra de 32 años, originaria de Dublín, Irlanda, comentaron que sus ginecólogos prescribían de manera sistemática la pastilla, sin tomar en cuenta los posibles efectos secundarios o sus propias inquietudes. Así, María afirmó: "las consultas siempre fueron muy rápidas y mis inquietudes nunca fueron tomadas en cuenta. Mi ginecóloga apenas mencioné la palabra "pastilla" me la prescribió inmediatamente. En lugar de advertirme acerca de los posibles efectos, me dijo que la pastilla era lo más maravilloso y que me iba a ayudar con el spm (Síndrome Premenstrual), con el cual nunca había tenido problemas y que a miles de mujeres les gustaba". Por su parte, Alicia comentó: "creo que los ginecólogos prescriben la pastilla de manera descontrolada, no se dan cuenta de que es una droga muy, muy poderosa. Nunca he escuchado a un médico decir que los efectos secundarios sean producidos por la pastilla. ¿Me tengo que morir primero para que lo admitan?".

En el caso de estas mujeres, la práctica médica aparece como desvalorizada. Alicia también hace hincapié en un elemento que nos parece importante: los médicos niegan que los síntomas experimentados por estas mujeres sean efectos secundarios provocados por la pastilla. Este último punto es importante, ya que pone en relieve un escenario que es generalizado entre los testimonios de las mujeres encuestadas, a saber, la minimización, por parte de los 
médicos, de la gravedad de los efectos secundarios producidos por la pastilla.

Existe por lo tanto, según nuestro criterio, un contexto en el cual las mujeres están "predestinadas" a sacrificar su salud y su bienestar en nombre de una contracepción "fiable". Esto crea una situación en la que las mujeres deben "probar" sus sufrimientos y sus problemas si no quieren que se les considere como "locas". Magaly, una estudiante de doctorado canadiense de 29 años, comentó: "a mí lo que me gustaría es que mi ginecólogo admita que esto es un problema hormonal y no que me lo estoy inventando". Por su parte, Verónica, una joven estadounidense de 25 años, afirmó: "cuando mencioné mis problemas, tres médicos distintos me dijeron que era imposible que fueran provocados por la pastilla. Busqué información en Google y el foro Aphrodite salió. Ahora sé que no estoy loca". A partir de estos testimonios, se nota una tensión entre las percepciones de los médicos y la percepción de las mujeres encuestadas. En efecto, la evaluación de los riesgos y de los efectos secundarios de la pastilla revela una diferencia de actitudes $y$ de percepciones, en cuanto a la utilización de los contraceptivos hormonales. Así, mientras los ginecólogos presentan los efectos secundarios como transitorios, las mujeres encuestadas los describen como efectos que perturban profundamente sus vidas.

Otro aspecto que las mujeres encuestadas encuentran problemático, es el hecho de que los ginecólogos no les advierten acerca de los posibles efectos secundarios. Son ellas mismas quienes, gracias a su propia iniciativa y experiencia, llegan a la conclusión de que la pastilla es la fuente de sus problemas de salud. Laura comentó: "lo que me gustaría es que los médicos advirtieran a las mujeres sobre los efectos secundarios. Yo no sabía que podía sufrir cambios de humor y depresión. Yo hice la conexión sola cuando la dejé de tomar y me di cuenta de que era la pastilla". Además, las mujeres encuestadas consideran que no solamente los médicos ignoran los posibles efectos secundarios de la pastilla, sino que deciden "enmascarar" esos efectos prescribiendo antidepresivos. Muchas de ellas se niegan a tomar los antidepresivos como una señal de resistencia. Victoria, por ejemplo, relató: "cuando me vi confrontada a una depresión, mi médico me recetó antidepresivos pero yo me rehusé tajantemente. Fue un largo año en donde aprendí mucho de mí misma”.

Esta resistencia, expresada en la insatisfacción generalizada experimentada por estas mujeres hacia sus ginecólogos, pasa también por una búsqueda de alternativas al saber médico oficial. Alicia, por ejemplo, dijo: "la verdad prefiero ir donde un naturalista. Siento que me escucha, toma en cuenta mis preocupaciones, mucho más que mi ginecóloga, que todavía insiste en que mis problemas son por estrés y no por la pastilla”. Así, es interesante subrayar cómo ciertos elementos del guión de género de la pastilla se vuelven problemáticos cuando las mujeres deben insertarse en un sistema médico que supone relaciones de poder verticales, en donde el saber de las mujeres sobre su propio cuerpo es constantemente negado y minimizado. Como veremos a continuación, este guión es también problemático en otro espacio de relaciones.

\subsection{LA PAREJA}

Las mujeres encuestadas, al decidir no utilizar la pastilla como método contraceptivo, debieron confrontarse a una negociación, a veces infructuosa, de las desigualdades sexuales $y$ reproductivas con sus compañeros. La mayoría de ellas expresaron una voluntad de querer compartir con los hombres la responsabilidad contraceptiva. Ante la posibilidad de utilizar otros métodos contraceptivos, especialmente el preservativo, las actitudes de los hombres estuvieron divididas. Se notó dos tendencias principales, por una parte, los hombres para los cuales el uso del preservativo era problemático. Victoria, por ejemplo, comentó cómo para su compañero, los preservativos no eran una opción. Para ella, la responsabilidad de la contracepción era su obligación: "cuando tomaba la pastilla, si me olvidaba de tomar una, mi novio se rehusaba a usar condón. Ahora tengo que ver qué método no hormonal utilizo porque no quiero volver a la pastilla". El caso de Laura es similar: "su principal preocupación era la pérdida de placer. Fue realmente 
imposible convencerlo. Ahora estamos usando el diafragma". Varios elementos son importantes de destacar a partir de los testimonios de estas mujeres. En efecto, el rechazo a usar preservativos por parte de los hombres refuerza dos discursos dominantes acerca de la sexualidad contemporánea. Primero, que la responsabilidad contraceptiva es femenina. Segundo, que el placer sexual masculino es de vital importancia. Estos dos principios constituyen la base de una forma de masculinidad dominante $y$ hegemónica (Oudshoorn, 2003) que refuerza las desigualdades sexuales $y$ reproductivas entre mujeres $y$ hombres.

Sin embargo, existe una contraparte a esta actitud. En efecto, otra tendencia que se observa entre los compañeros de las mujeres encuestadas, revela otra forma de masculinidad que se aleja del modelo de masculinidad hegemónica descrito anteriormente. Es el caso de Gloria, una estudiante inglesa de 19 años, cuyo compañero no tenía ningún problema en utilizar preservativos: "mi novio me decía que él prefería usar dos condones antes que verme de nuevo tomando las pastillas. ¡El decía que mis depresiones eran horribles! Hablaba de las hormonas como de las "horrormonas" y así les digo desde entonces, porque son horribles". Por otro lado, Isabel, una mujer irlandesa de 35 años, dijo que ella decidió dejar de tomar las pastillas en gran parte gracias a su esposo: "mi marido me insistió tanto para que dejara las pastillas con el fin de poner un punto final a mis problemas de salud". De la misma forma, María afirmó: "mi novio estaba muy contento de que yo dejara las pastillas porque me quería ver de nuevo tranquila y con buena salud. La verdad, no creo que la pastilla masculina sea algún día una opción. Además, no podría confiar en él porque de todos modos, no es él el que se embaraza".

María también hace alusión a otro tema: una posible pastilla masculina ${ }^{7}$. Se debe señalar que si bien, ella está dispuesta a delegar la responsabilidad de la contracepción (su compañero usa preservativos), no es favorable a

$7 \quad$ Para un análisis detallado acerca de las razones por las cuales la pastilla masculina no se ha desarrollado, ver Oudshoorn, 2003. la idea de una pastilla masculina porque no podría confiar en que los hombres efectivamente la tomen. Este elemento es interesante porque remite a la idea de que los hombres, de todos modos, no tendrían la motivación suficiente para encargarse por ellos solos de la contracepción, idea que de hecho, es un aspecto importante del discurso sobre la masculinidad hegemónica. En el caso de María, el preservativo es preferible porque así ella puede controlar si lo está usando o no, lo cual sería más difícil en el caso de una pastilla. Si bien, ella delega la responsabilidad de la contracepción, es ella quien controla la situación.

Con respecto a la pastilla masculina, las mujeres se mostraron dubitativas. Es el caso por ejemplo, de Katia: "no sé qué pensar. Por un lado, me parecería extraordinario que hubiera una nueva opción contraceptiva. Por otro lado, no puedo evitar pensar que lo que sufrí yo, lo puede sufrir mi novio. Además, no sé si los hombres sean muy receptivos a la idea de una pastilla para ellos".

A partir de los testimonios de este segundo grupo de mujeres, se analiza que las actitudes de los hombres con respecto a la posibilidad de compartir la responsabilidad contraceptiva, es diferente en relación a los compañeros de las mujeres del primer grupo. En ese sentido, algunas mujeres lograron (re)negociar de una mejor forma las desigualdades sexuales y reproductivas, logrando resistirse al guión que pone sobre ellas toda la responsabilidad de la contracepción y a reconfigurar las relaciones de género al interior de la pareja. Sin embargo, la mayoría de las mujeres encuestadas se mostraron reticentes a la idea de una pastilla masculina, lo que revela las limitaciones culturales que todavía pesan sobre la idea de un posible contraceptivo hormonal masculino (Oudshoorn, 2003). A continuación, se analiza otro espacio donde la resistencia y la búsqueda de alternativas parecieron evidentes: el foro Aphrodite.

\subsection{APHRODITE}

En el caso de las mujeres encuestadas, Internet parece ser un verdadero espacio alternativo donde ellas esperan encontrar respuestas a las preguntas e inquietudes que como 
se observó anteriormente, el sistema médico oficial parece no ofrecerles. En ese sentido, los foros funcionan como una especie de espacio público, en el cual las mujeres pueden discutir directamente con otras mujeres y así compartir sus experiencias. Convertirse en miembra de Aphrodite, reviste de una importancia muy particular. En efecto, para las mujeres encuestadas, participar de la vida de este foro es la expresión de una forma de resistencia colectiva. Así, el intercambio de experiencias individuales constituye en su conjunto, una especie de sabiduría colectiva que le propone a las mujeres modos de resistencia a los guiones de género analizados en las secciones anteriores.

De esta manera, participar en el foro Aphrodite es concebido por estas mujeres como un mecanismo de apoyo y de validación. Al escribir en el foro, ellas expresan una voluntad no solamente de compartir sino también de encontrarle un sentido a sus propias experiencias. Para estas mujeres, ser miembra del foro se convirtió, en una táctica de sobrevivencia a los efectos secundarios de la pastilla. Carmen, por ejemplo, una ama de casa inglesa de 39 años, contó cómo la conexión con otras mujeres representó un mecanismo para aprehender mejor su propia situación: "yo diría que este foro es uno de los elementos más importantes para entender lo que pasa con nuestros cuerpos. Además, se obtiene el apoyo moral necesario y en general, información que los médicos no nos dan. Cuando dejé las pastillas estaba aterrorizada, pero pude entender todo gracias a esta comunidad de damas". Para Carmen, las experiencias de las otras mujeres miembras del foro constituyen una fuente central de saberes acerca de sus propios cuerpos. Se puede resaltar que esos saberes se conciben como paralelos a las instancias tradicionales de producción del conocimiento médico, es decir, son saberes concebidos por las mujeres para las mujeres. Así, el foro se convierte en un espacio en el cual, las mujeres cuestionan el saber y la práctica médicos oficiales. María, por ejemplo, afirmó: "creo que este tipo de plataformas son muy importantes porque es el único medio donde mujeres como yo se sienten tomadas en serio". Para María el foro es un lugar en donde se validan sus experiencias.

De la misma manera, el foro es también un espacio en donde las mujeres aspiran a otro estilo de ejercicio de la medicina. Violeta, por su parte, comentó: "me hice miembra tratando de encontrar alternativas no hormonales porque mi médico no hacía más que recetarme la pastilla, el implante o el dispositivo intrauterino. Quería saber qué estaban usando otras mujeres". Otro elemento central es el hecho de que el intercambio colectivo de las experiencias individuales, es un medio para estas mujeres se den cuenta que la culpabilidad psicológica ("me estoy volviendo loca") respaldada por los médicos, es estructuralmente producida y puede ser resistida. Roxana, una enfermera canadiense de 32 años contó: "comencé en este foro porque pensé que me estaba volviendo loca. Me ayudó mucho hablar con mujeres que habían tenido experiencias similares. Es importante tener una especie de verificación de que una no se está imaginando cosas". Para Roxana, el participar en el foro es un mecanismo de resistencia a la idea de que las malas experiencias de las mujeres con la pastilla son imaginarias. Para otras mujeres, la voluntad de compartir experiencias se traduce en una voluntad por educar a otras mujeres, en un verdadero activismo que sobrepasa los límites virtuales del foro. Katia compartió: "siempre hablo de lo que me pasó con mis amigos y familiares. Además, envío cartas a periódicos, revistas, programas de televisión $y$ hablo también con los hombres. Le dedico mucho tiempo a esto. El foro es extremadamente importante en todo esto". A partir del testimonio de Katia, parece interesante notar que ella le atribuye un lugar importante al foro entre sus prácticas activistas. Este punto es central en la medida en que el foro puede ser concebido como un espacio en donde lo privado se vuelve público. En ese sentido, el foro puede ser pensado como una verdadera herramienta de emancipación.

Por lo tanto, el foro Aphrodite es percibido como un espacio público abierto, en el cual las mujeres encuestadas pueden aportar un testimonio directo y en la mayoría de los casos, sumamente detallado, de sus sufrimientos $y$ 
frustraciones en cuanto a la pastilla. Este intercambio de experiencias representa para estas mujeres, una oportunidad de develar su intimidad, de contribuir a la interacción del grupo, así como de desplegar las situaciones vividas, las cuales con frecuencia, son desvalorizadas por sus médicos ginecólogos. De esta manera, las mujeres adquieren un sentido de autonomía y de reconocimiento de sí mismas como expertas, que les son negados en el seno del sistema médico oficial.

En ese sentido, este cuestionamiento se traduce en una voluntad por reconocer sus propias experiencias $y$ las de otras mujeres como fuentes legítimas de conocimiento sobre sus propios cuerpos. Así, el intercambio colectivo de experiencias $y$ de conocimientos constituye una forma colectiva de resistencia, en la medida en que estas mujeres intentan borrar la rígida frontera que separa el saber del experto $y$ el saber del no-especialista (Löwy, 2005). Los saberes elaborados y compartidos por las mujeres del foro constituyen una especie de poder.

\section{CONCLUSIONES}

El análisis de los testimonios de las mujeres que participaron en este estudio, demostró que la pastilla - la mayoría de las veces descrita como una herramienta de emancipación- es cuestionada, puesto que para este grupo de mujeres, el hecho de no consumirla representa una liberación. En efecto, estas mujeres expresan una resistencia a la pastilla que se materializa en sus vidas cotidianas en un conjunto de tensiones, tentativas de negociación y búsqueda de alternativas con otros actores sociales implicados en su consumo, en particular, con sus médicos ginecólogos, sus compañeros sentimentales y las otras mujeres miembras del foro Aphrodite. Sin embargo, parece importante precisar que los resultados de este análisis son representativos del grupo estudiado.

Este análisis no pretende ser una crítica a la pastilla, ya que se reconoce que existen millones de mujeres que deben a esta tecnología el alivio de dolores menstruales insoportables $y$ una gran tranquilidad en cuanto al riesgo de un embarazo no deseado. En ese sentido, la historia de la pastilla es una historia a la vez de liberación y de control. Por un lado, la pastilla es probablemente la tecnología contraceptiva más eficaz jamás concebida. Como contraceptivo, constituye una herramienta de emancipación para una gran cantidad de mujeres. Por otro lado, el objetivo con este artículo es hacer evidente la necesidad de aprehender la ciencia $y$ las tecnologías contraceptivas, en todas sus tensiones $y$ ambigüedades.

Las experiencias y los testimonios de las mujeres encuestadas fueron analizados a la luz de un enfoque constructivista y feminista de los estudios de la ciencia y la tecnología, los cuales conciben la ciencia como una práctica histórica, social y cultural. Por lo tanto, se parte de la idea de que la producción científica se desarrolla en el seno de una sociedad bien determinada, la cual condiciona sus objetivos, los agentes $y$ sus modos de funcionamiento. Siguiendo este razonamiento, se considera que la pastilla es un guión de género, es decir, una tecnología que materializa en sí misma representaciones, valores y prácticas de género muy particulares.

En ese sentido, se concibe el consumo de la pastilla como una performance de ciertas convenciones de género, a saber, la hormonización de los cuerpos femeninos, la biomedicalización, la estandarización y la regulación de los ciclos menstruales, la imposición de la responsabilidad contraceptiva y reproductiva, así como de los riesgos asociados a la contracepción hormonal y finalmente, la institucionalización de ciertas prácticas médicas patriarcales.

Este control social ejercido por medio de la pastilla es contestado por la mayoría de las mujeres encuestadas. En efecto, para este grupo de mujeres, el consumo de la pastilla no representa una forma de emancipación sino más bien una forma de dominación. La resistencia a esta dominación se revela difícil y paradójica para estas mujeres, asimismo se manifiesta visiblemente en espacios sociales en donde ciertas tensiones, negociaciones $y$ alternativas convergen. En cada uno de estos espacios, se constató que las mujeres encuestadas se sirven de diferentes mecanismos para intentar resistir, (re)negociar o buscar alternativas a los guiones de género materializados en la pastilla. Se concluye que esta resistencia se traduce 
en una redefinición más o menos exitosa, de ciertos elementos de sus identidades de género $y$ de su feminidad.

Así, será importante para esta autora continuar este tipo de estudios realizando un análisis comparativo de las prácticas contraceptivas de dos países diferentes por determinar. Las preguntas que guiarían tal análisis serían las siguientes: ¿cuáles son los métodos contraceptivos más utilizados en esos dos países?, ¿qué implicaciones tienen para las mujeres que los utilizan?, ¿podemos identificar resistencias?, ¿podemos ubicar esas resistencias en la historia actual de las medicinas alternativas $y$ de todo un movimiento de cuestionamiento de la biomedicina que no concierne solo a las mujeres y sus cuerpos? Tales son las preguntas a las cuales se pretende contestar en una futura investigación.

\section{BIBLIOGRAFÍA}

Akrich, Madeleine y Méadel, Cécile. "Problématiser la question des usages". Sciences Sociales et Santé 22 (1). 2004: 5-20.

Boellstorff, Tom. Coming of age in second life: an anthropologist explores the virtually human. Princeton: Princeton University Press, 2008.

Burrell, Jenna. "The field site as a network: a strategy for locating ethnographic research". Field Methods 21 (2). 2009: 181-199.

Butler, Judith. Gender trouble. Feminism and the subversion of identity. New York: Routledge, 1990.

Butler, Judith. Bodies that matter. On the discursives limits of "sex". New York: Routledge, 1993.

Cuevas, Tania. "Tecnologías contraceptivas y feminidad: la pastilla como un script de género". Revista Reflexiones, en prensa.

Clarke, Adele E. Disciplining reproduction: modernity, american life sciences, and the problems of sex. Berkeley: University of California Press, 1998.

Coleman, E. Gabriella. "Ethnographic approaches to digital media”. Annual review of anthropology 39. 2010: 1-16.
Department of Economics and Social Affairs. Population Division, United Nations. 2012 Update for the MDG Database: Contraceptive Prevalence. 2012. En: $<w w w . u n p o p u l a t i o n . o r g>$.

Engelman, Peter C. A history of the birth control movement in America. Santa Barbara, California: Praeger, 2011.

Ortiz Gómez, Teresa. "Interacciones entre salud, historia y feminismo. Una revisión historiográfica”. Diálogo filosófico 59. 2004: 229-244.

Hine, Christine. Virtual ethnography. London: SAGE, 2000.

Kline, Ronald. "Resisting consumer technology in rural America: the telephone and electrification". How users matter. The co-construction of users and technology. Nelly Oudshoorn y Trevor Pinch (eds.). Cambridge, MA. MIT Press, 2003: 51-66.

Laqueur, Thomas W. Making sex: body and gender from the Greeks to Freud. Cambridge, Mass: Harvard University Press, 1990.

Löwy, Ilana. "Le féminisme a t-il changé la recherche biomédicale? Le women health movement et les transformations de la médécine aux états-unis". Travail, genre et sociétés 2 (14). 2005: 89-108.

Moscucci, Ornella. The science of woman: gynecology and gender in England, 1800-1929. Cambridge: Cambridge University Press, 1990.

Orgad, Shani. "Moving from online to offline relationships with research participants". Virtual Methods. Christine Hine (ed.). Oxford: Berg, 2005.

Oudshoorn, Nelly. "On the making of sex hormones: research materials and the production of knowledge". Social Studies of Science 20 (1). 1990: 5-33.

Oudshoorn, Nelly. Beyond the natural body. An archeology of sex hormones. London: Routledge, 1994.

Oudshoorn, Nelly. "Clinical trials as a cultural niche in which to configure the gender identities of users: the case of the male contraceptive development". How users matter. The co-construction of users and 
technology. Nelly Oudshoorn y Trevor Pinch (eds.). Cambridge, MA. MIT Press, 2003: 209-228.

Pinch, Trevor y Bijker, Wiebe. "The social construction of facts and artefacts or how the sociology of science and the sociology of technology might benefit each other". The social construction of technological systems: new directions in the Sociology and History of technology. Wiebe Bijker, Terry Hughes y Trevor Pinch (eds.). Cambridge, Mass: MIT Press, 1987.

Pincus, Gregory. "Progestational agents and the control of fertility". Vitamins and Hormones 169. 1959: 307-325.

Rogers, Richard. Digital Methods. Cambridge, MA: MIT Press, 2013.

Sinding, Christiane. "Le sexe des hormones: L'ambivalence fondatrice des hormones sexuelles". Cahiers du Genre 34. 2003: 39-56.

Thomsen, Steven R.; Straubhaar, Joseph y Bolyard, Drew. "Ethnomethodology and the study of online communities: exploring the cyber streets". Information Research 4 (1). 1998.

Tone, Andrea. Devices and desires: a history of contraceptives in America. New York: Wang and Hill, 1999.
Toulemon, Laurent y Leridon, Henri. "Vingt années de contraception en France: 1968-1988". Population 46 (4). 1991: 777-811.

Van Oost, Ellen. "Materialized gender: how shavers configure the users' femininity and masculinity". How users matter. The co-construction of users and technology. Nelly Oudshoorn y Trevor Pinch (eds.). Estados Unidos: Masachusetts Institute of Technology Press, 2003.

Walstrom, Mary K. "Ethics and engagement in communication scholarship: analyzing public, online support groups as researcher/participant-experiencer". Virtual Research Ethics: Issues and Controversies. Elisabeth A. Buchanan (ed.). Hershey, pa. Information Science Publishing, 2004: 174-202.

Wyatt, Sally. "Non-users also matter: the construction of users and non-users of the internet". How users matter. The co-construction of users and technology. Cambridge, ма. міт Press, 2003: 67-80.

Fecha de ingreso: 06/03/2013 Fecha de aprobación: 09/07/2013 
